\title{
La máquina eólica para desaguar terrenos pantanosos de Agustín de Betancourt y Molina: análisis de su construcción mediante ingeniería asistida por ordenador
}

\section{Agustin de Betancourt's wind machine for draining marshy ground: analysis of its construction through computer-aided engineering}

$\underline{\text { J.I. Rojas-Sola }}^{(*)}$, E. de la Morena-de la Fuente ${ }^{(* *)}$

\section{RESUMEN}

El objetivo de esta investigación ha sido analizar la construcción de la máquina eólica para desaguar terrenos pantanosos diseñada por Agustín de Betancourt y Molina en 1789. Para ello, se ha realizado un análisis estático por elementos finitos a partir del modelo tridimensional obtenido con Autodesk Inventor Professional. Los resultados muestran que las mayores tensiones del mecanismo se producen cuando el eje principal está engranado con la corona dentada, concretamente, en el punto de contacto entre el tornillo sinfín y dicha corona. Sin embargo, los máximos desplazamientos y las mayores deformaciones tienen lugar en las palas impulsoras. Asimismo, el mecanismo está sobredimensionado, no alcanzando en ningún punto la tensión de rotura del material, confirmando la acertada construcción de esta invención histórica.

Palabras clave: Agustín de Betancourt; máquina eólica para desaguar terrenos pantanosos; diseño asistido por ordenador; ingeniería asistida por ordenador; análisis estático; análisis por elementos finitos; Autodesk Inventor Professional.

\section{ABSTRACT}

The objective of this research is to analyze the construction of the wind machine for draining marshy ground designed by Agustin de Betancourt and Molina in 1789. To do this, a static analysis by finite elements method from the threedimensional model obtained with Autodesk Inventor Professional has been performed. The results show that the greatest stresses of the mechanism take place when the main shaft is meshed with the cogwheel, namely the point of contact between the worm screw and cogwheel. However, the maximum displacements and the greatest deformations take place in the blades. In addition, the mechanism is oversized, reaching at no point the tensile strength of the material, confirming the successful construction of this historical invention.

Keywords: Agustin de Betancourt; wind machine for draining marshy ground; computer-aided design; computeraided engineering; static analysis; finite element analysis; Autodesk Inventor Professional.

(*) Escuela Politécnica Superior de Jaén, Universidad de Jaén (España).

$\left({ }^{* *}\right)$ Programa de Doctorado, Universidad de Córdoba (España).

Persona de contacto/Corresponding author: jirojas@ujaen.es (J.I. Rojas Sola)

ORCID: http://orcid.org/oooo-0001-9001-1050 (J.I. Rojas Sola); http://orcid.org/oooo-0003-1407-5903

(E. de la Morena-de la Fuente)

Cómo citar este artículo/Citation: Rojas-Sola, J.I., de la Morena-de la Fuente, E. (2018). La máquina eólica para desaguar terrenos pantanosos de Agustín de Betancourt y Molina: análisis de su construcción mediante ingeniería asistida por ordenador. Informes de la Construcción, 70(549): e236. https://doi.org/10.3989/id.54738

Copyright: (c) 2018 CSIC. Este es un artículo de acceso abierto distribuido bajo los términos de la licencia de uso y distribución Creative Commons Reconocimiento 4.0 Internacional (CC BY 4.0). 


\section{INTRODUCCIÓN}

La figura de Agustín de Betancourt y Molina como insigne ingeniero español (1758-1824) está fuera de toda duda con notables aportaciones a diferentes ámbitos de la ingeniería, en particular en la construcción de obras de ingeniería civil, aparte de ser uno de los creadores de la Teoría de las Máquinas y Mecanismos junto a José María Lanz y Zaldívar (17641839).

Existen un gran número de publicaciones que abarcan aspectos biográficos (1) (2) (3) (4) (5) (6), y otros relativos a su obra científica y técnica (7) (8) (9). Por ello, conscientes de la relevancia de su obra, la Fundación Canaria Orotava de Historia de la Ciencia puso en marcha el proyecto 'Betancourt' (10), disponiendo en su página web de una sección con toda la información, y además, de libre acceso para cualquier usuario.

Esta original e inédita investigación forma parte de una tesis doctoral, y continúa otra publicación realizada con anterioridad sobre el modelado tridimensional con técnicas de diseño asistido por ordenador (CAD, del inglés Computer-Aided Design) de la invención objeto de estudio (11), concretamente de la máquina eólica para desaguar terrenos pantanosos diseñada en Francia 1789, por el ingeniero canario.

Así pues, el principal objetivo es realizar un estudio de ingeniería asistida por ordenador (CAE, del inglés ComputerAided Engineering) a partir del modelo CAD 3D, concretado en un análisis estático por elementos finitos con indicación de las tensiones de von Mises, desplazamientos, deformaciones equivalentes y coeficiente de seguridad, gracias al concurso del software Autodesk Inventor Professional, para analizar la idoneidad de la construcción de dicha invención y su integridad, siguiendo de esta manera la línea de investigación abierta sobre ejemplos notables del patrimonio histórico de sobresalientes personajes españoles.

La importancia de esta tesis doctoral radica en que no existen estudios desde el punto de vista de la ingeniería de ninguna de las aportaciones de su obra, tan sólo un estudio desde la ingeniería gráfica del telégrafo óptico de Betancourt (12), y de ahí, su originalidad, novedad y conveniencia.

\section{MATERIAL Y MÉTODOS}

El material original de partida ha sido el disponible en el sitio web del proyecto digital Betancourt (13), y consiste únicamente en una lámina con las vistas diédricas, detalles y perspectiva caballera, y otra lámina con detalles a mayor escala del sistema rotor y del sistema regulador de llenado de artesas.

\subsection{Diseño asistido por ordenador}

Como se ha indicado, el modelo CAD $3 \mathrm{D}$ se ha obtenido gracias al concurso del software paramétrico Autodesk Inventor Professional 2016 (14), realizando una restitución digital a partir de la escasa información disponible en el expediente tanto descriptiva como planimétrica, lo que ha obligado a asumir una serie de hipótesis dimensionales y geométricas para que el funcionamiento de la invención fuera el correcto. La Figura 1 muestra un plano de conjunto con indicación de todas las marcas (componentes) de la invención.
A partir de la Figura 1, se va a describir su funcionamiento. La máquina eólica para desaguar terrenos pantanosos consta de dos partes fundamentales: por un lado, está el sistema rotor impulsor (basado en el molino eólico de eje vertical de origen persa), y por otro, el mecanismo de desagüe. Dicho eje vertical [2] se mueve por la incidencia del viento en las palas [3] como en los molinos de eje vertical. La gran diferencia con ellos es el sistema ideado por Betancourt para conseguir adaptar su invento ante condiciones de viento realmente escasas aprovechando la energía eólica de un modo más eficaz. Mientras que en los molinos de eje vertical es necesario evitar la incidencia del viento en la mitad de las palas para conseguir que el sistema rotor gire, el ingeniero español propone un sistema nuevo que evita una estructura compleja y expuesta a esfuerzos adicionales.

La solución que aporta Betancourt es permitir que las palas estén unidas a sus guías [1] por un eje excéntrico de modo que puedan girar libremente sobre dicho eje. Con esta sencilla operación, las palas de la máquina eólica se dispondrían todas en la dirección del viento como si se tratara de una veleta, oponiendo al viento la menor resistencia posible. Para que esto no sucediera en todas las palas a la vez, Betancourt dispone en las guías unos topes de modo que impidan que la pala pueda girar libremente $360^{\circ}$. Así pues, cuando el viento soplara en una dirección, las palas girarían libremente presentando la menor resistencia a viento, y dos de ellas chocarían contra el tope de la guía provocando el giro de todo el sistema, y por tanto, haciendo que rotara el eje principal de transmisión (Figura 2).

El extremo de dicho eje está apoyado en una pieza de madera denominada carro [9] que presenta un grado de libertad; de este modo, el sistema rotor impulsor no sólo gira sino que además puede desplazarse a derecha e izquierda (Figura 3). Asimismo, formando parte del mismo eje, y en su extremo, hay un tornillo sinfín [11] que gira solidariamente con el eje. Ésta va a ser la manera de transmitir el movimiento al mecanismo de desagüe.

El mecanismo de desagüe está sostenido en un bastidor de madera que forma parte de la estructura principal [4] que soporta todas las tensiones estructurales. De un lado, facilita que el eje principal siempre esté en posición vertical, y por otro lado, aloja todo el mecanismo elevador de agua. La base del bastidor es un cuadrado por el que ascienden y descienden dos cubetas a modo de cangilones a la zona inundada.

Así pues, se parte de una posición en la que el eje principal está pegando a uno de los tambores [5]. Al estar en esa posición, la corona dentada que está en el extremo del tambor [10] se encuentra engranada con el tornillo sinfín de dicho eje, de manera que al girar el eje por acción del viento, esto provoca que el tornillo sinfín y la corona giren solidariamente. El movimiento del tambor se transmite a la cuerda que está enrollada una sola vez en el zona media del tambor. La cuerda está dispuesta de modo que está enrollada enambos tambores y de sus extremos se suspendan las dos cubetas, una llena (la que sube), y otra vacía (la que desciende). El movimiento del otro tambor se debe a dos motivos: de una parte, en su extremo hay una cubeta que pesa, y de otra, la cuerda que une ambas está tirante, lo que permite el movimiento. Al girar el tambor, la parte de cuerda que está entre ambos tambores crece pero el peso de la cubeta vacía hace que gire descendiendo la otra. 


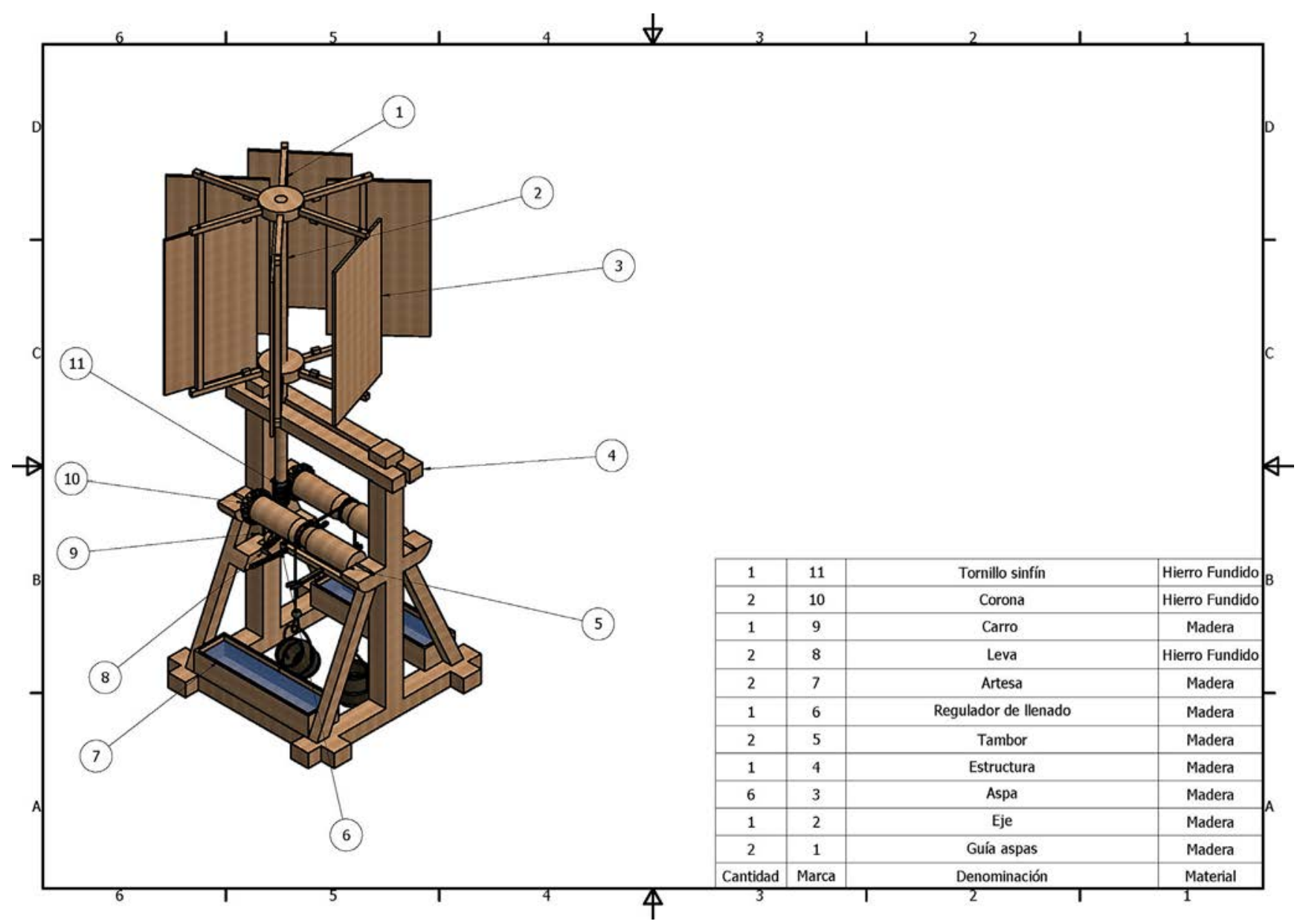

Figura 1. Plano de conjunto de la invención con todos sus componentes.
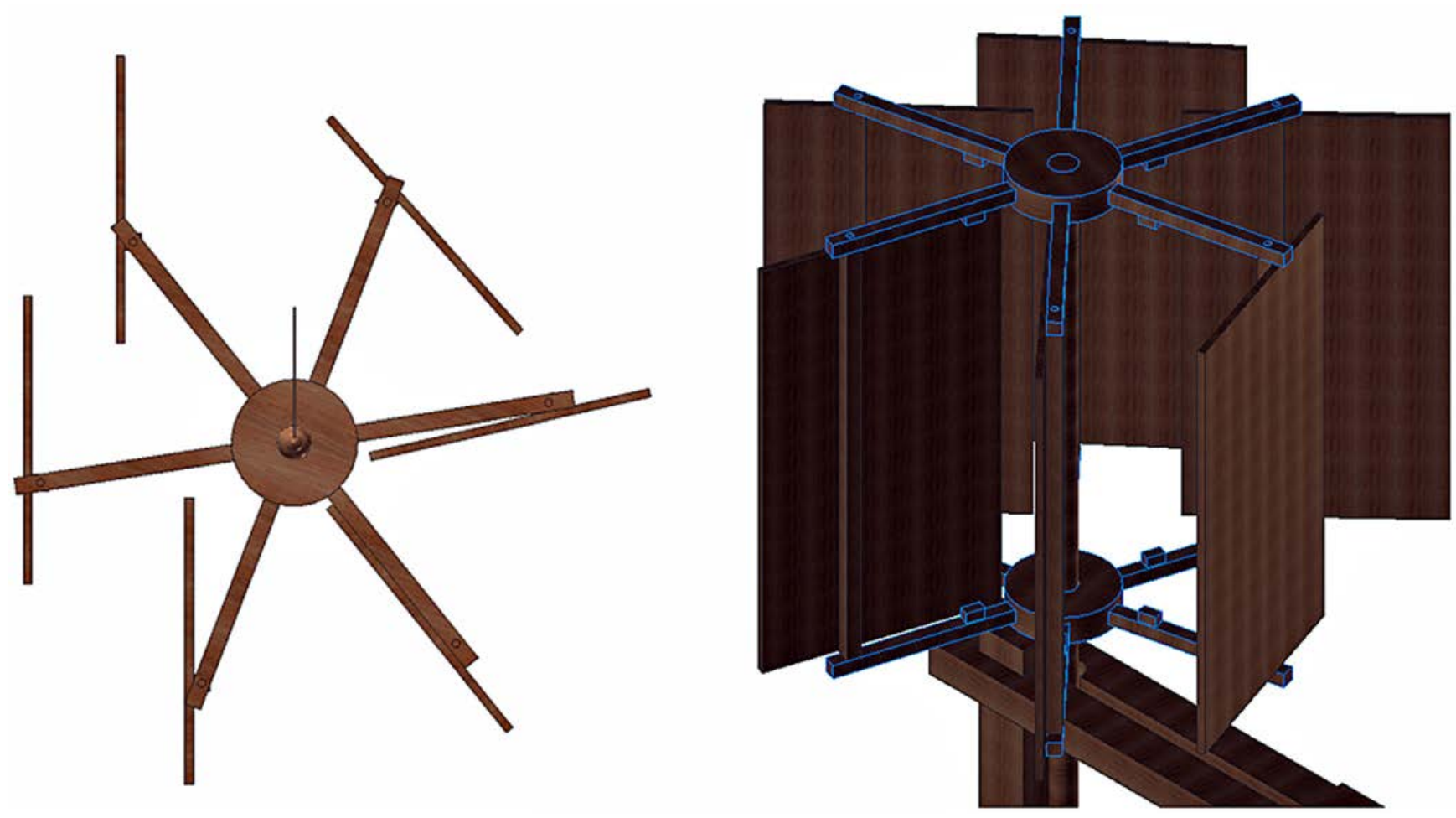

Figura 2. Vista en planta del sistema rotor que provoca el movimiento del conjunto (Izda.) y detalle de las guías que soportan y hacen de tope a las palas (Dcha.). 
Sobre el asa de la cubeta llena de agua hay una esfera metálica que funciona a modo de tope. En determinado momento, ésta choca con el regulador de llenado [6], y hace que cambie su posición balanceándolo de su posición a la de equilibrio en el otro extremo. El regulador de llenado es un eje horizontal con diversas extensiones laterales a modo de balancines, de modo que al girar dicho regulador levanta la leva [8] que libera el movimiento del carro que sostiene el extremo del eje vertical. El carro es empujado por un martillete inserto en el regulador de llenado lo que provoca que el tornillo sinfín se desengrane moviéndose lateralmente hasta chocar con la rueda dentada del otro tambor. Durante este tiempo, el mecanismo de desagüe se para. Al engranar el otro tambor con el tornillo sinfín, éste empieza a girar en sentido contrario y la cubeta que descendía asciende ahora (supuestamente cargada de agua). De este modo, van ascendiendo alternativamente una $\mathrm{u}$ otra cubeta desaguando paulatinamente la zona inundada. La cubeta en su movimiento ascendente choca con una vara de hierro que le obliga a girar volcando el líquido sobre las artesas [7] confinadas en la parte inferior de la estructura. Las artesas presentan una tubería de desagüe en dirección a un lugar donde alojar el agua.

La originalidad del sistema reside tanto en el modo en cómo el mecanismo impulsor aprovecha la energía eólica con alta eficiencia como en el sistema de alternancia del movimiento ascendentes-descendente de las cubetas.

\subsection{Ingeniería asistida por ordenador}

\subsubsection{Preprocesado}

Antes de realizar la simulación del análisis estático del mecanismo conviene establecer una serie de simplificaciones que faciliten su ejecución sin que por ello el resultado del mismo se vea afectado. De este modo, las necesidades de cálculo serán menores y el análisis más fácil de realizar.

- Se han eliminado las cubetas, cuerdas y demás elementos del sistema de elevación sustituyéndolos por dos momentos en cada uno de los tambores. Todos estos elementos serán sustituidos por un momento de la fuerza que provoca el peso del agua cargada por la cubeta sobre el tambor cilíndrico. Asimismo, se han despreciado el peso del asa, de los materiales pequeños y de la misma cuerda porque son valores pequeños, y porque del otro tambor cuelgan los mismos elementos actuando a modo de contrapeso.

- Se ha eliminado la veleta de la estructura superior. Este elemento ofrece una información sobre la dirección del viento pero no tiene ninguna función estructural y su peso es despreciable.

- Se va a suponer que las artesas están completamente llenas de agua, lo que se va a traducir en dos presiones determinadas en el fondo de cada una de ellas, ya que el análisis de tensiones se va a realizar en el caso más desfavorable.

- Se ha despreciado el peso de las levas frente al resto de tensiones ocasionadas, ya que además no tienen ninguna función estructural, por lo que se eliminan del análisis.

Con lo dicho hasta aquí, cabe concluir que el mecanismo de estudio será el formado por dos grandes conjuntos: de una parte, la estructura superior formada por palas (aspas), sus guías y eje vertical con su tornillo sinfín en el extremo inferior, y de otra, por la estructura inferior formada por el bastidor, los dos tambores cilíndricos con su coronas metálicas, el carro que facilita el movimiento lateral del eje vertical, y por último el balancín de llenado.

Queda por exponer una última condición que va a cambiar sustancialmente el resultado del análisis, y es que la posición que tenga el eje vertical de la máquina no es indiferente. No resulta lo mismo que el tornillo sinfín trabaje engranado con las coronas de los tambores o que no lo haga. Por ello, se va a realizar el estudio de tensiones teniendo en cuenta dos posiciones extremas: en la primera de ellas, el eje estará engranado con los tambores, y en la segunda, el eje descansará completamente en el carro (Figura 3).

\subsubsection{Asignación de materiales}

Una vez que se ha pre-procesado el modelo asumiendo las simplificaciones indicadas previamente, hay que asignar a cada elemento del ensamblaje un material concreto con unas determinadas propiedades. La escasísima información que se ha obtenido sobre la invención histórica estudiada no permite conocer con exactitud, los materiales empleados por el ingeniero canario en su construcción. Aun así, se han asignado materiales semejantes a los que pudo tener acceso Betancourt, y en particular, de la biblioteca de materiales del software utilizado (Autodesk Inventor Professional), se han empleado aquellos que se ajustaban a los requerimientos operativos, es decir, hierro fundido para piezas metálicas y roble para las de madera, que por otro lado es el material principal de la máquina.

Las propiedades térmicas, mecánicas y los límites de resistencia de estos materiales se pueden consultar en el citado mismo software. El hierro fundido tiene un comportamiento isótropo y sus propiedades físicas principales son: módulo de Young (210.00o MPa), coeficiente de Poisson (o,30), densidad $\left(7.150 \mathrm{~kg} / \mathrm{m}^{3}\right)$ y tensión de rotura $(758 \mathrm{MPa})$. En cambio, la madera de roble tiene unas propiedades más complicadas ya que es un material ortótropo. Se sabe que si se actúa en la dirección de la veta, las propiedades son mejores pero no así, en las otras dos direcciones. Por tanto, se ha supuesto que la dirección de la veta es la que define la dirección principal de los cuerpos, como es lógico, y por lo tanto, sus propiedades físicas son: módulo de Young (210.00o MPa), coeficiente de Poisson $(0,30)$, densidad $\left(760 \mathrm{~kg} / \mathrm{m}^{3}\right)$ y tensión de rotura (41 MPa).

\subsubsection{Condiciones de contorno}

Una vez que se tienen aplicados los materiales a todos los componentes del conjunto, el siguiente paso en la preparación de la simulación es la de imponer condiciones de contorno. Cada uno de los componentes del conjunto puede funcionar de determinada manera atendiendo a su movilidad, lo que afectará al análisis estático del ensamblaje.

En primer lugar, se tienen elementos que tienen una restricción de movimiento completa, como son los componentes fijos. Así pues, en este caso, la base de la estructura principal se considera empotrada o fijada al suelo (Figura 4 (Izda.)).

Un segundo tipo de restricción, con un grado más de libertad, son aquellos componentes que no pueden desplazarse longitudinalmente pero sí pueden girar; en Autodesk Inventor Professional, esta restricción se llama de rodadura o de 

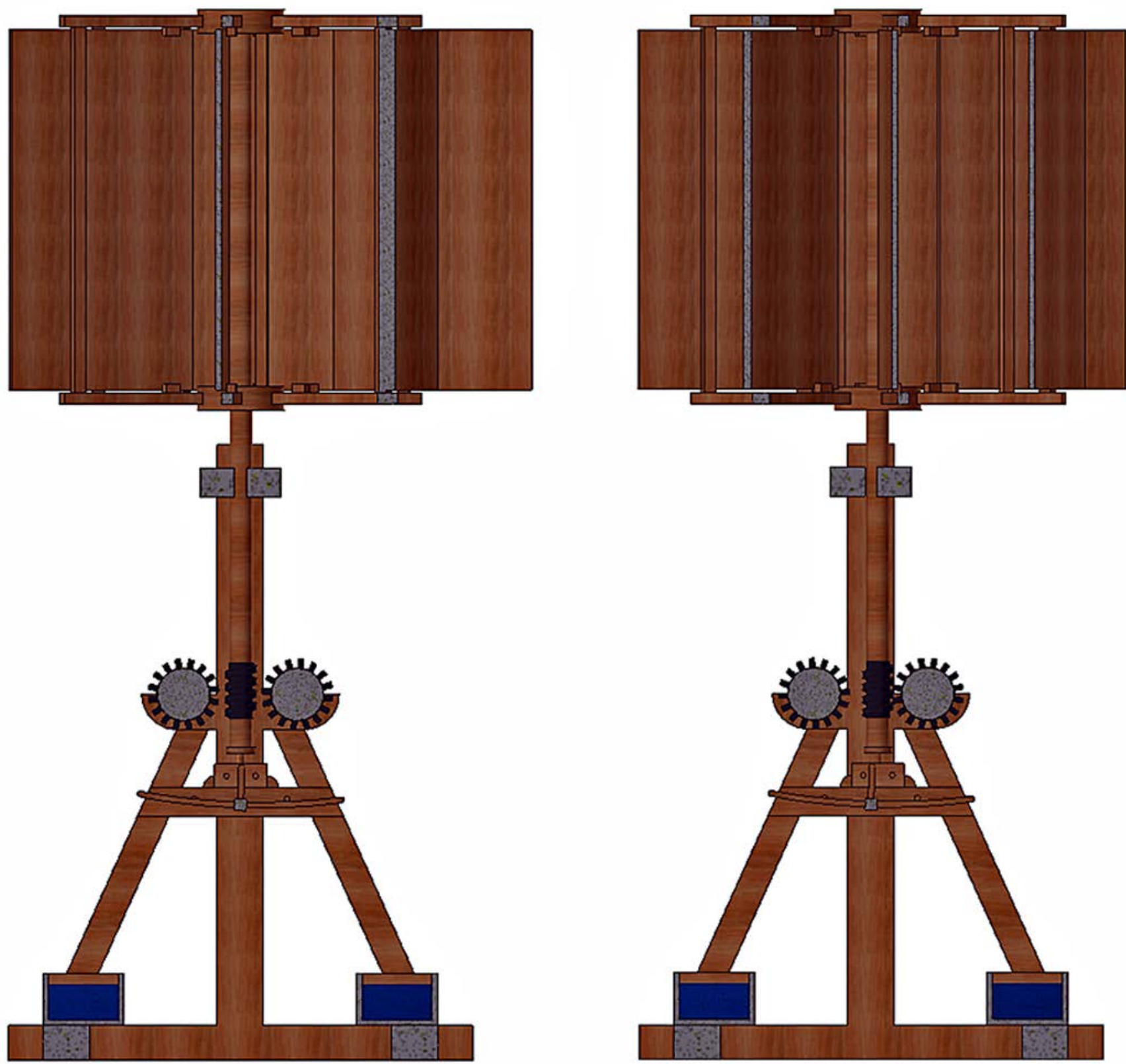

Figura 3. Posición centrada (Izda.) y posición engranada (Dcha.).

pasador, presentándola un buen número de componentes. Así pues, en primer lugar existe una restricción entre el eje vertical y la base o carro donde se apoya; en segundo lugar, entre las aspas y las guías, y por último, el de los elementos horizontales (tambores cilíndricos, levas y regulador de llenado) apoyados en el bastidor inferior con los tambores y el mecanismo de llenado (Figura 4 (Dcha.)).

Un tercer tipo de restricción es el que presenta un grado de libertad más, deslizante o sin fricción, y es el que se establece entre el carro que soporta el peso del eje principal y el bastidor (Figura 4 (Inferior)).

Por último, es necesario indicar que las condiciones de contorno no se ven afectadas por las dos posiciones anteriormente citadas aunque sí que hay que definir los contactos correctamente. Así pues, para que el análisis de tensiones sea correcto hay que activar los contactos entre todas las piezas del ensamblaje; de este modo Autodesk Inventor Professional determina automáticamente las superficies entre las dis- tintas piezas que van a entrar en contacto. No obstante, el software permite definir manualmente algunos contactos en el caso de que por su complicada geometría no sea capaz de identificarlos correctamente. Éste es el caso del contacto que existe entre el eje vertical y el taladro practicado en la parte superior del bastidor, y cuya función es mantener la posición vertical del eje (Figura 5). Asimismo, a la hora de estudiar la posición engranada, se debe definir manualmente otro contacto importante, y es el que se establece entre el tornillo sinfín y la corona dentada del tambor. El resto de piezas tienen correctamente definidos sus contactos.

\subsubsection{Fuerzas aplicadas}

La siguiente etapa en el análisis estático del conjunto, es concretar la acción de las cuatro fuerzas que van a incidir sobre él: la acción del viento sobre las palas, el peso del agua sobre el fondo de las artesas, las fuerzas sobre los tambores producida por el agua elevada por la cubeta y, por último, la acción de la gravedad sobre todo el conjunto (Figura 6). 

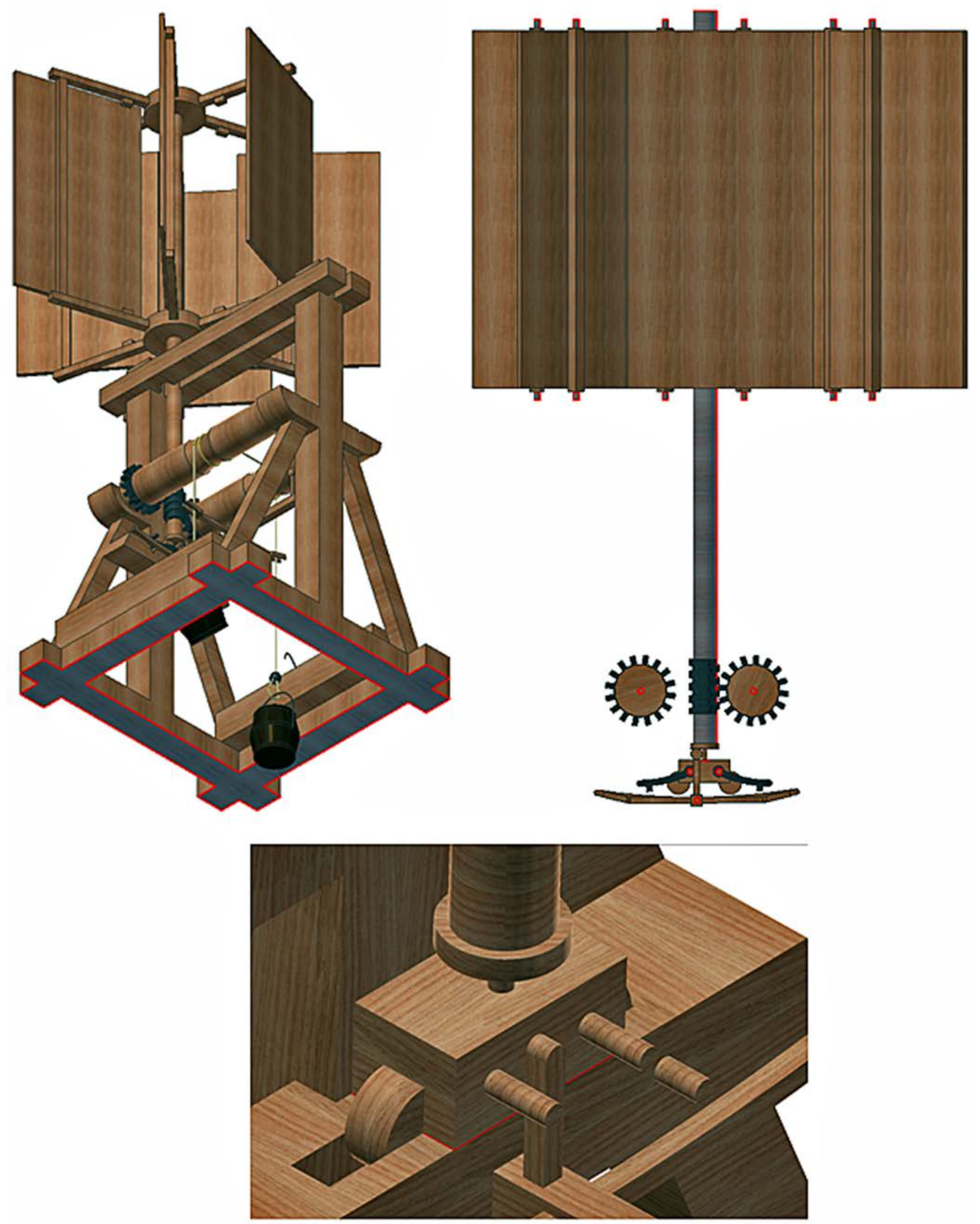

Figura 4. Tipos de restricciones: Fija (Izda.), Rodadura (Dcha.) y sin fricción (Inferior).

La presión del viento ejercida sobre las palas es la fuerza principal que acciona la invención, de ahí la necesidad de su correcto cálculo. Sin embargo, la incidencia del viento sobre las palas es un estudio que requeriría un análisis exhaustivo desde la diná- mica de los fluidos. Por ello, para el presente estudio se va a simplificar su incidencia, ya que el objetivo de este estudio es estructural, de manera que se va a calcular la incidencia del viento en función de la densidad del aire y la velocidad del viento. 

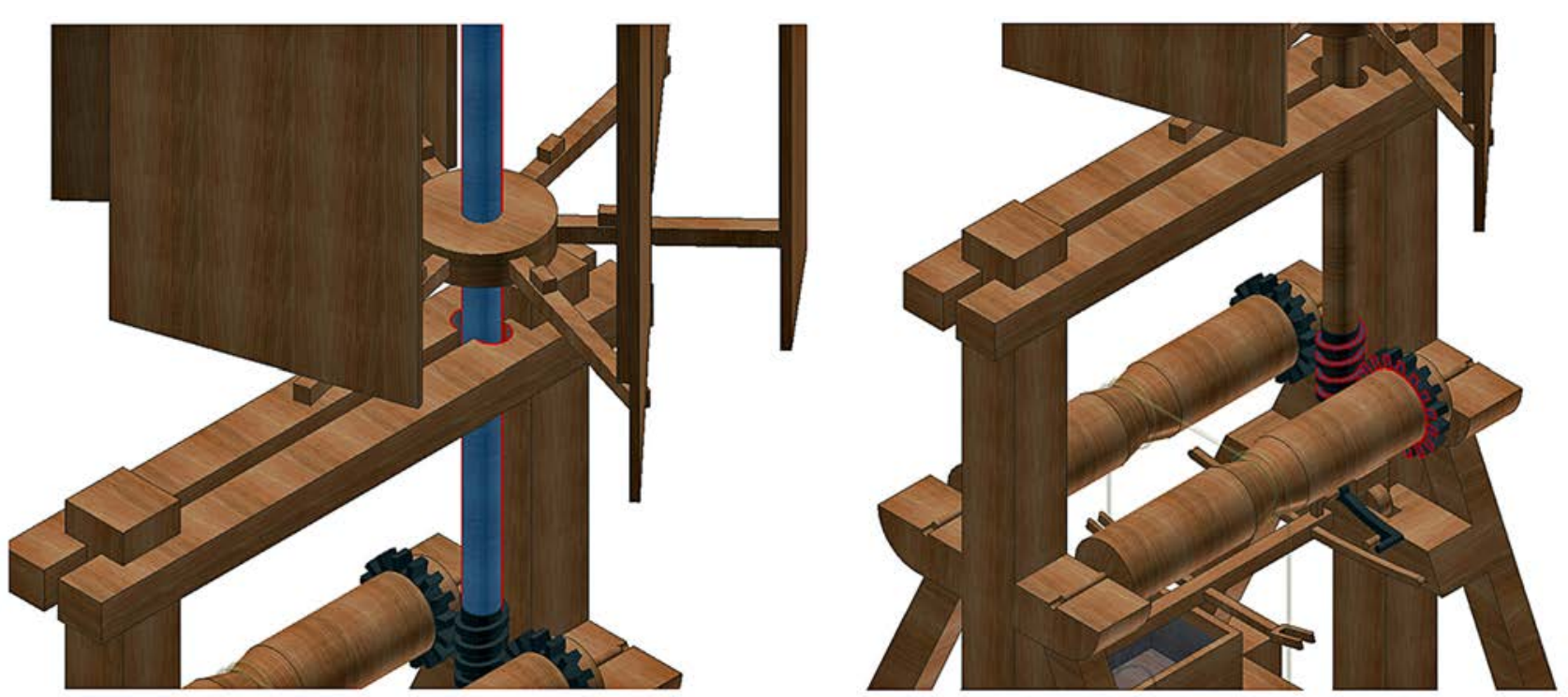

Figura 5. Activación de contactos de forma manual.

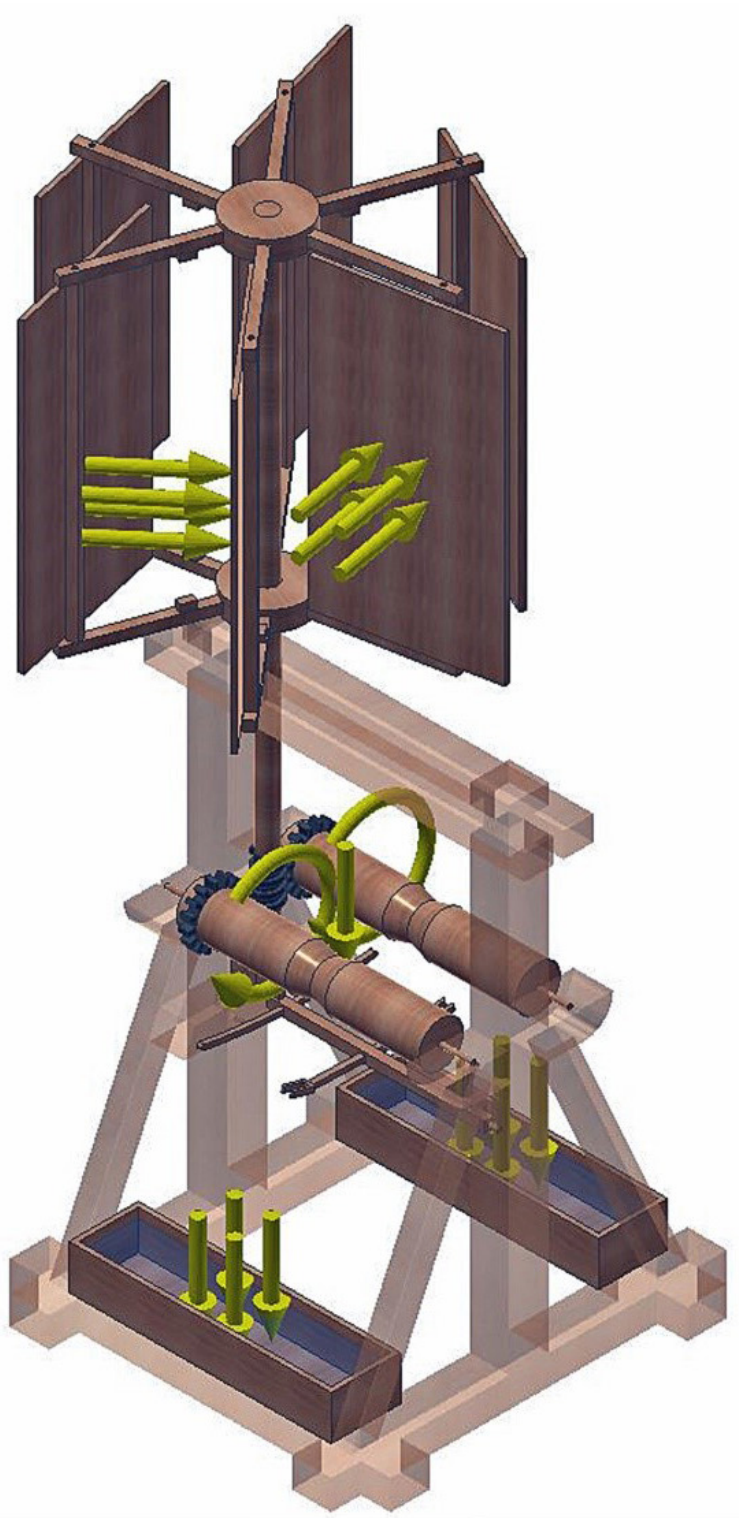

Figura 6. Fuerzas que inciden en el modelo: debido al aire (aspas), al peso del agua (artesas), al peso de las cubetas (momentos en los tambores) y a la gravedad (aplicada al centro de gravedad del mecanismo)
Para obtener la velocidad del viento, se va a suponer que la máquina eólica trabajó en algún momento en algún lugar de Francia donde Betancourt trató de ponerla en funcionamiento. Para ello, acudiendo al Eurocódigo 1 (norma que especifica la incidencia del viento para ser considerada en el cálculo de estructuras y edificaciones) y en su Anexo A, se puede observar que presenta valores por zonas con valores comprendidos entre los 24 y $34 \mathrm{~m} / \mathrm{s}$ (15).

Los valores de velocidad de viento son muy altos para unas hipotéticas condiciones de trabajo. En esas condiciones la máquina eólica debería permanecer parada. De todas maneras para el presente análisis se va a tomar el menor de los valores, es decir, el correspondiente a $24 \mathrm{~m} / \mathrm{s}$, condición realmente extrema que nunca sería la de trabajo.

La energía cinética (Ec) del viento que incide sobre las palas corresponde con la expresión siguiente:

$$
E c=\frac{1}{2} m v^{2}
$$

Siendo $m$, la masa que transmite una cantidad determinada de energía, y $v$, la velocidad de dicha cantidad de aire. Además, se sabe que la masa que transmite la energía tiene esta expresión donde $\delta$ es la densidad del aire y $\mathrm{V}$ el volumen de aire que comunica esa energía:

$$
m=\delta \cdot V
$$

Sustituyendo en la ecuación de la energía cinética se obtiene:

$$
E c=\frac{1}{2} \delta V v^{2}
$$

Por otro lado, la presión ( $\sigma$ ) que ejerce el aire será el cociente entre la energía cinética (Ec), y el volumen de aire que la contiene (V), de manera que sustituyendo las expresiones anteriores, se tendrá que:

$$
\sigma=\frac{E c}{V}=\frac{\delta v^{2}}{2}=\frac{1}{2} \cdot \frac{1,293 \mathrm{~kg}}{\mathrm{~m}^{3}} \cdot \frac{24^{2} \mathrm{~m}^{2}}{\mathrm{~s}^{2}}=372,40 \mathrm{~Pa}
$$


Así pues, la presión que ejerce el aire por unidad de superficie de la pala es de 372,40 Pa.

Como se puede apreciar en la Figura 2, el aire realmente sólo incide de manera eficaz en dos de las palas. El resto de palas tiene una alineación paralela a la dirección del aire por lo que no presentan oposición. Así pues, se supone que el viento actúa sólo sobre esas dos palas. Las palas, de todos modos no siempre son perpendiculares a la dirección del viento, y de hecho cuando esto ocurre, la que es perpendicular resta mucho aire a la siguiente. Así pues, se propone un coeficiente de corrección para ambas palas. Se considera un coeficiente de corrección de 0,85 para la primera pala (la de mayor incidencia) y 0,5 para la segunda. Pese a que estos coeficientes son arbitrarios no dejan de ser una aproximación bastante realista. Así pues, se tendrán las siguientes presiones:

$$
\begin{aligned}
& \sigma_{1}=0,85 \cdot \sigma=316,53 \mathrm{~Pa} \\
& \sigma_{2}=0,5 \cdot \sigma=186,20 \mathrm{~Pa}
\end{aligned}
$$

La segunda de las fuerzas a modelizar es la del peso del agua de las artesas. Su cálculo es mucho más sencillo porque el agua está modelizada aunque después se haya eliminado del análisis de tensiones para hacer más sencillo su cálculo. El software Autodesk Inventor Professional proporciona las propiedades físicas de cada uno de los componentes modelizados, por lo que se conoce el volumen del agua de cada artesa de valor $0,459 \mathrm{~m}^{3}$, su masa (m) con un valor de 485,22 kg, así como el área (A) del fondo de la artesa de valor $1,53 \mathrm{~m}^{2}$. Con estos datos se calcula de una manera sencilla la presión sobre el fondo de la artesa. La ecuación de la presión $(\sigma)$ es:

$$
\sigma=\frac{\mathrm{F}}{\mathrm{A}}=\frac{\mathrm{m} \cdot \mathrm{g}}{\mathrm{A}}=\frac{485,22 \mathrm{~kg} \cdot 9,81 \mathrm{~m} / \mathrm{s}^{2}}{1,53 \mathrm{~m}^{3}}=3.111,12 \mathrm{~Pa}
$$

Donde g es la aceleración de la gravedad. Así pues, en la condición más desfavorable la presión que el agua ejerce sobre el fondo de cada una de las artesas cuando están llenas de agua, es de 3.111,12 Pa, que sería la condición más desfavorable de cara a un análisis estático.

La siguiente fuerza a modelizar es la producida por el agua ascendida por la cubeta. Como se ha explicado anteriormente, el peso del agua ascendida incide directamente en los tambores en los que está enrollada la maroma que asciende y desciende las cubetas. Se ha sustituido este peso por un momento de fuerza aplicado por igual en cada uno de los tambores. Ese momento se calcula a partir del peso que asciende de la cubeta.

Para su cálculo, se conoce que el momento de fuerza es el producto vectorial entre la Fuerza (F) y el radio de giro (r) de del cuerpo sobre el que se aplica la fuerza:

$$
\vec{M}=\vec{F} \times \vec{r}
$$

La fuerza de la que se trata es el peso de la cubeta llena de agua, que tal y como se ha indicado anteriormente, es calculado automáticamente por el software al revisar las características (iProperties) del agua de la cubeta. Se sabe que el volumen de dicha cubeta es de 100 litros, lo que equivale a
979,04 N de peso, y el radio de 0,225 m. Sustituyendo en la fórmula, se tiene que:

$$
|\vec{M}|=979,04 \mathrm{~N} \cdot 0,225 \mathrm{~m}=220,28 \mathrm{Nm}
$$

Existe un momento de fuerzas en cada uno de los tambores de 220,28 Nm que tiene que vencer el tornillo sinfín. Si la fuerza del viento no es capaz de superar este momento la cubeta no subirá el agua. Así pues, se puede suponer que uno de los puntos donde habrá una solicitación mayor será precisamente aquel donde tocan el tornillo sinfín y la corona dentada del tambor. Hay que precisar que cuando el eje está en una posición centrada, la cubeta está vacía, y por lo tanto, este momento no existe para dicha posición.

Por último, falta por incluir la gravedad en la simulación. Para incluir esta magnitud basta con definirla. Para ello el software permite representar el vector aplicado en el centro de gravedad del mecanismo $\left(9,81 \mathrm{~m} / \mathrm{s}^{2}\right)$ en sentido negativo del eje $\mathrm{Z}$.

Finalmente, una vez aplicadas todas las fuerzas, la simulación queda preparada a expensas de la discretización del dominio computacional a considerar y posterior cálculo.

\subsubsection{Discretización o mallado}

El último paso antes de la simulación es discretizar todo el ensamblaje a partir de un mallado que debe ajustarse lo mejor posible a la geometría real del modelo. Los pasos que se van a dar a continuación van encaminados, precisamente, a conseguir una malla lo más fidedigna posible a la realidad modelada. Sin embargo, los puntos donde es especialmente importante ser cautos con la generación automática que nos propone el software son dos: en primer lugar, en aquellos lugares en los que la geometría es más compleja, y en segundo, en los puntos o regiones donde se aplican las fuerzas.

Al configurar el cálculo automático de la malla hay que determinar una serie de variables. El software propone automáticamente un tamaño medio para la malla del $10 \%$ de la longitud del elemento a discretizar, un tamaño mínimo del elemento del 20\% del tamaño medio, un factor de modificación con valor de 1,5, y un ángulo máximo de giro de $60^{\circ}$. Sin embargo, estos factores se pueden modificar para determinar una malla más acorde con la geometría del mecanismo.

El presente trabajo ha utilizado los valores por defecto de mallado pero sí ha realizado un mallado especial para los elementos más sensibles, citados anteriormente. Para ello Autodesk Inventor Professional permite realizar un control de malla local seleccionando la superficie sobre la que se quiere hacer un refinamiento del mallado y proponiendo un nuevo tamaño de la malla en mm. Así pues, para las zonas sensibles se ha establecido un tamaño de elemento de $15 \mathrm{~mm}$ consiguiendo de este modo una malla variable pero más adaptada a la geometría de los objetos. El control de malla local se ha utilizado fundamentalmente en el punto de apoyo del eje vertical con el carro, en la inserción de las palas con sus guías, en el punto del contacto del eje vertical con el taladro practicado en el bastidor y en el fondo de las artesas. La Figura 7 muestra un ejemplo de cómo varía el mallado respecto al proporcionado en la discretización automática. 


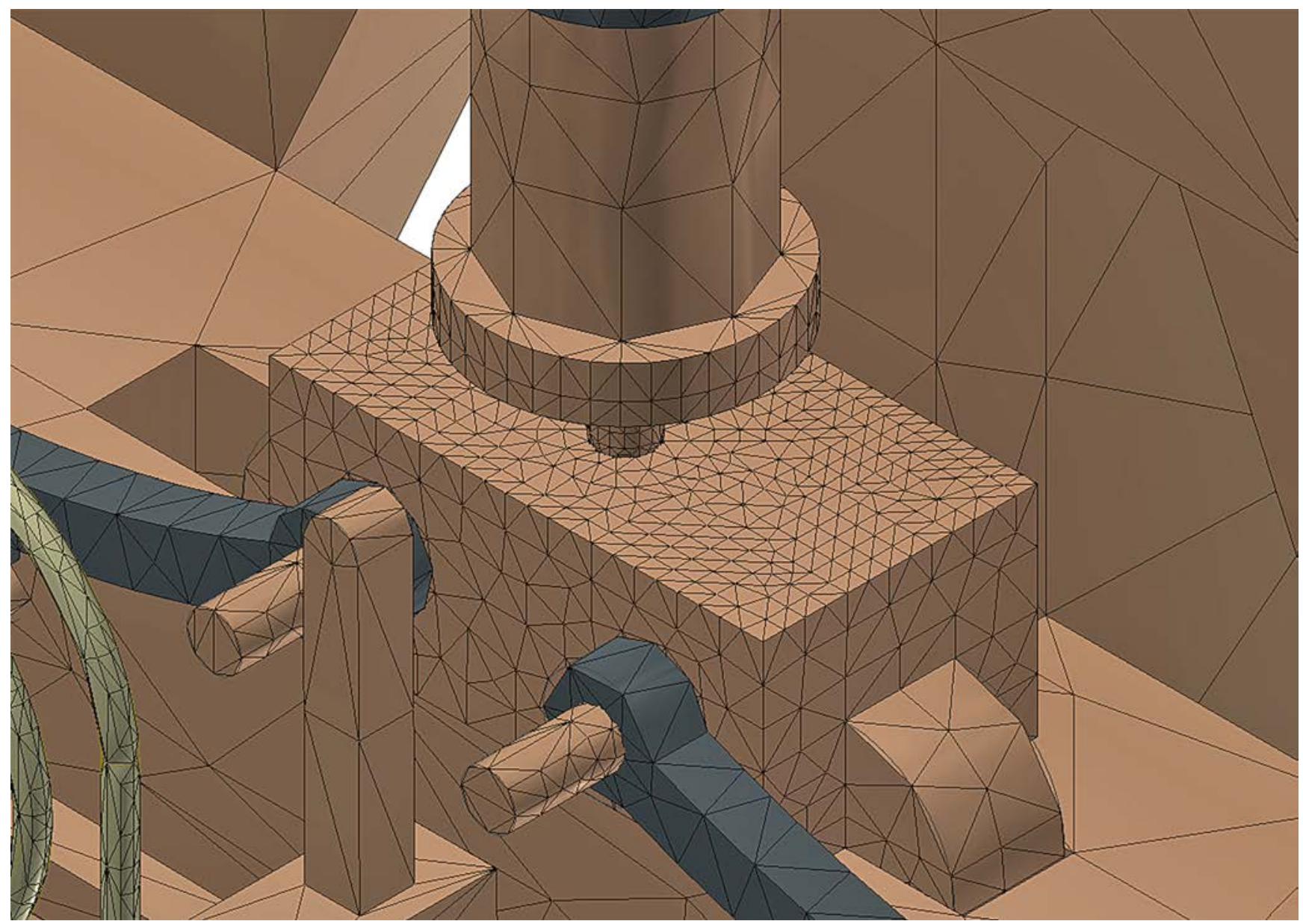

Figura 7. Discretización obtenida tras el refinamiento de la malla en el apoyo del eje.

\section{RESULTADOS Y DISCUSIÓN}

El análisis estático realizado por el software proporciona resultados sobre las tensiones de von Mises, los desplazamientos, las deformaciones y el coeficiente de seguridad.

Como ya se comentó anteriormente, hay que diferenciar el estudio de las tensiones teniendo en cuenta las dos posiciones (centrada y engranada), ya que ambas posiciones van a arrojar resultados diferentes. El análisis de la tensión de von Mises (Figura 8) revela que el mecanismo está sometido por lo general a tensiones bajas, aunque hay algunos puntos singulares donde se dan puntualmente máximos que deben ser valorados.

Cuando el mecanismo está centrado hay tres puntos con valores altos de tensión: en las guías de las palas (en las inserciones con las palas y el eje), en el punto donde tocan el eje vertical y el taladro del bastidor, y por último, en el punto de apoyo del eje sobre el carro. La tensión con valor más alto (7,84 MPa) está localizada en el punto de contacto entre el eje vertical y la guía superior.

Por otro lado, cuando el mecanismo está engranado existe un punto singular, además de los citados anteriormente, y es precisamente, el punto de contacto entre el tornillo sinfín y la corona dentada del tambor cilíndrico, como cabía esperar. La tensión con valor más alto $(28,74 \mathrm{MPa})$, en este caso cambia, y está localizada precisamente, en este último contacto entre el tornillo sinfín y la corona dentada (Figura 9).
Por otro lado, se conoce que la tensión de rotura para compresión en una dirección paralela a la veta de la madera de roble es de $41 \mathrm{MPa}$, y la del hierro fundido $758 \mathrm{MPa}$. Los resultados obtenidos en el análisis de tensiones de von Mises, muestran que ningún punto del mecanismo supera la tensión de rotura; concretamente se conoce que el punto del mecanismo que tiene un mayor valor de 28,74 MPa es de hierro fundido, por lo que está lejos de dicho límite. Para que este resultado se observe gráficamente mejor, se puede cambiar la escala de colores poniendo como límite superior la tensión de rotura de la madera o del hierro fundido; sin embargo, en las imágenes mostradas se ha elegido un límite superior mucho más bajo (5 ó $10 \mathrm{MPa}$ ) con el fin de resaltar mejor las zonas con mayores esfuerzos.

Otra forma de conocer el estado de los materiales es calcular el coeficiente de seguridad que tiene en cuenta cada material independientemente y su deformación. El coeficiente de seguridad se obtiene como la relación entre el límite de elasticidad del material y la tensión de von Mises en cada punto. $\mathrm{Si}$ el coeficiente de seguridad es inferior a 1 significa que, en ese punto, la seguridad del mecanismo está comprometida, al alcanzar el límite de elasticidad. Un resultado aceptable sería un coeficiente de seguridad entre 2 y 4 . En el mecanismo estudiado, el punto con un coeficiente de seguridad menor $(5,5)$ es el punto de inserción del eje vertical con la guía de las palas. Por lo tanto, todo el mecanismo trabaja por encima del límite elástico del material, pero a la vez está lejos de trabajar en el rango óptimo. Se podría decir que el mecanismo, como era habitual en la época, estaba sobredimensionado y que se 

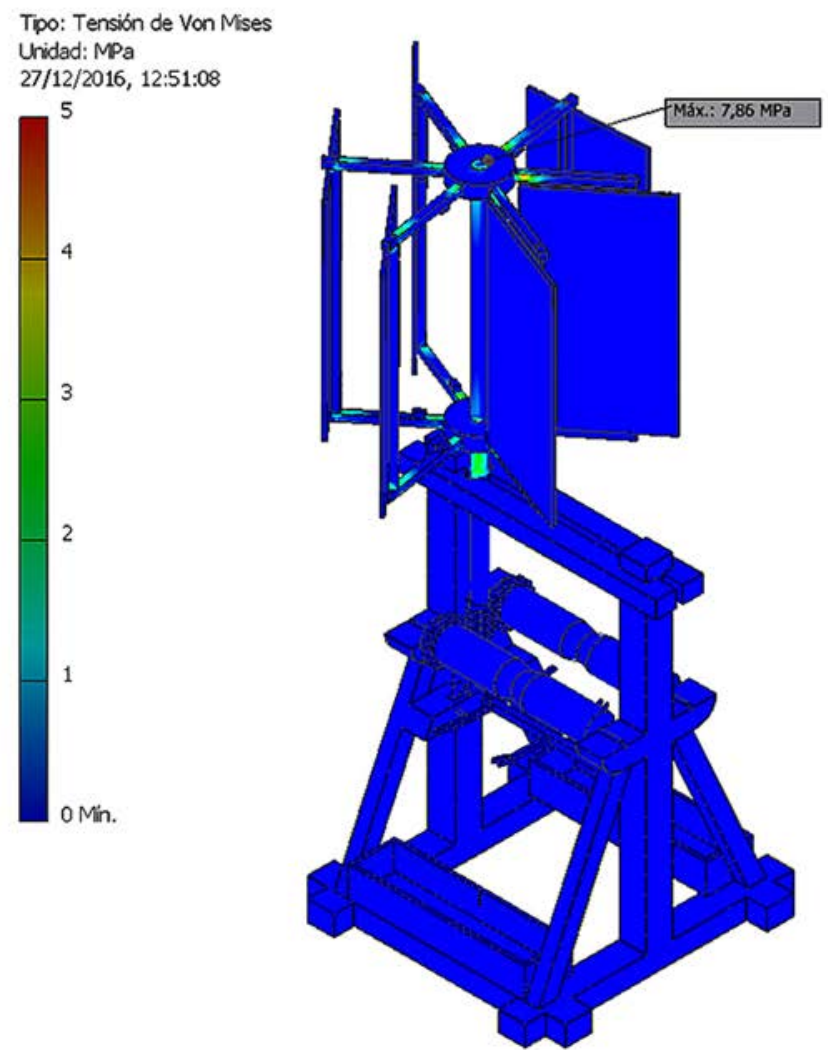

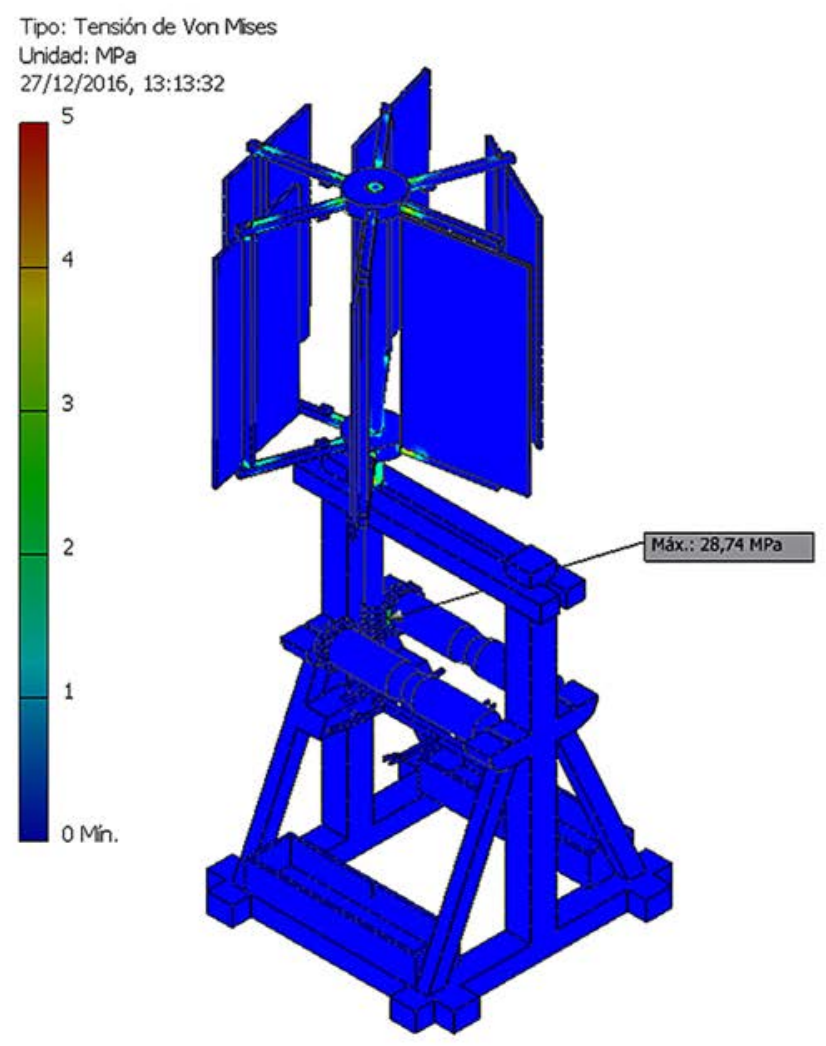

Figura 8. Tensiones de von Mises en la posición centrada (Izda.) y en la posición engranada (Dcha.).

Tipo: Tensión de Von Mises

Unidad: $\mathrm{MPa}$

27/12/2016, 12:22:30
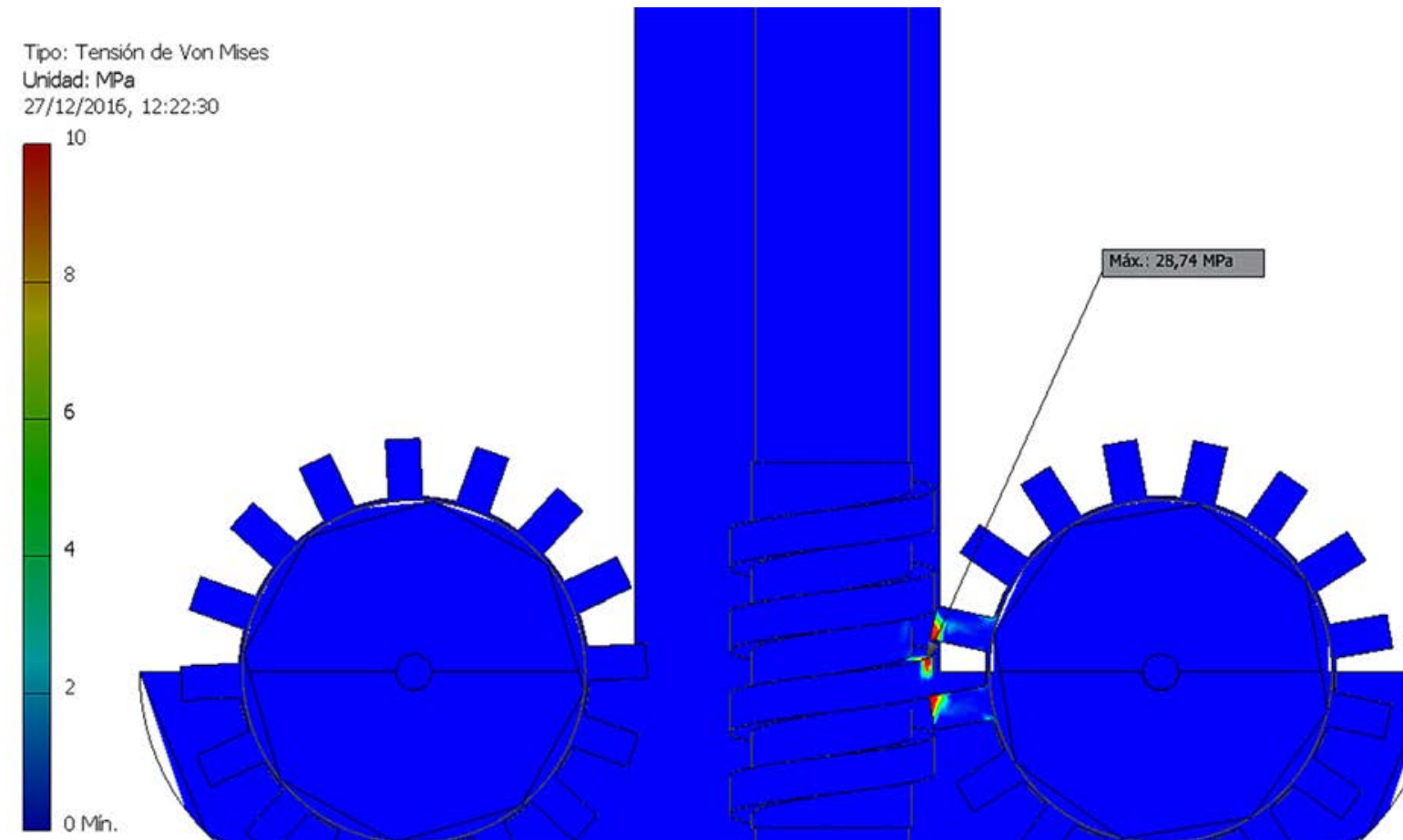

Min.
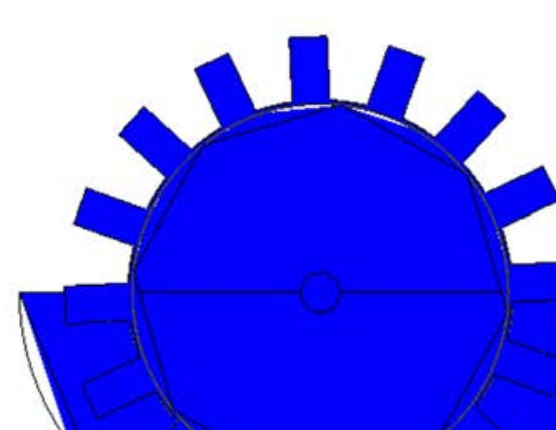

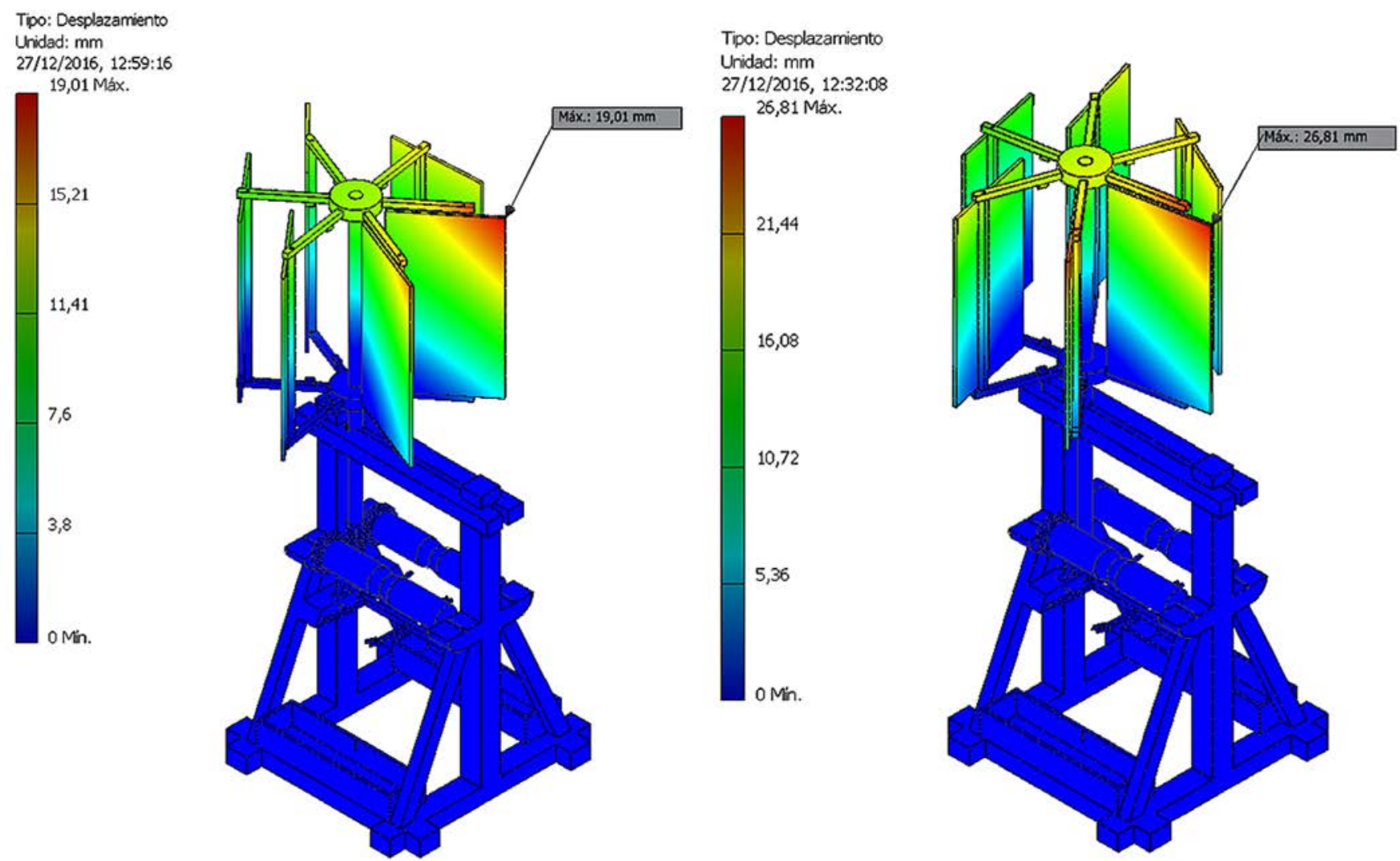

Figura 10. Distribución de desplazamientos en la posición centrada (Izda.) y en la posición engranada (Dcha.).

podría haber optimizado de cara a un mejor aprovechamiento de las propiedades de los materiales empleados.

Asimismo, las deformaciones equivalentes son distintas dependiendo de si el eje está engranado o no lo está. Cuando está en una posición centrada (sin engranar), la deformación mayor se produce en el extremo superior del eje vertical, pero cuando está engranado con la corona dentada, la deformación mayor se localiza en el punto de contacto del eje con el bastidor. En ambos casos, la deformación resulta muy pequeña.

Finalmente, se puede comprobar que los mayores desplazamientos se producen en el extremo de las palas sobre las que incide directamente el viento con independencia de la posición en la que se encuentre el eje. La posición del eje sólo va a influir en la magnitud del desplazamiento. En cualquier caso, el desplazamiento máximo es de $26,81 \mathrm{~mm}$, un desplazamiento asumible para un elemento flexible como la madera (Figura 10).

\section{CONCLUSIONES}

La presente investigación expone los resultados del análisis estático mediante técnicas de ingeniería asistida por ordenador (CAE) de la máquina eólica para desaguar terrenos pantanosos diseñada por Agustín de Betancourt y Molina en 1789. Para ello, se ha partido del modelo $\mathrm{CAD}_{3} \mathrm{D}$ realizado con Autodesk Inventor Professional, software paramétrico de diseño e ingeniería asistidos por ordenador.

El alto número de componentes, así como la complejidad de los mismos, ha justificado la simplificación del conjunto a analizar. Dicha simplificación, que no afecta a los resultados del análisis estático realizado, permite que el volumen computacional sea menor, y por tanto, reduce los altos requerimientos computacionales. Sin estas simplificaciones, los resultados obtenidos no hubieran mejorado y el tiempo de simulación hubiera sido mucho mayor. Sin embargo, la simulación de cuerdas y cables por parte de dicho software, es una asignatura pendiente.

Las mayores tensiones que soporta el mecanismo tienen lugar cuando éste está engranado con la corona dentada (ascendiendo agua), concretamente el punto de contacto entre el tornillo sinfín y dicha corona. Sin embargo, los máximos desplazamientos y las mayores deformaciones tienen lugar en la parte superior del mecanismo, es decir, en las palas impulsoras, como cabía esperar. En cualquier caso, de acuerdo con los resultados que arroja el estudio de los coeficientes de seguridad, el mecanismo está claramente sobredimensionado, lejos de aprovechar correctamente las características de los materiales empleados, y por otra parte, este modo de proceder era el común en los ingenios de la época, ya que no existían todavía ensayos con materiales.

Así pues, dicha invención de la que no se conoce nada sobre su ejecución material, fue un ingenio perfectamente proyectado: sobredimensionado y por tanto, diseñado para trabajar sin problemas estructurales ni mecánicos, y con unos requerimientos tanto de funcionamiento (necesidades eólicas) como de mantenimiento muy bajos. Por lo tanto, a partir de la presente investigación, se puede confirmar que su construcción pudo ejecutarse perfectamente.

\section{AGRADECIMIENTOS}

Esta investigación ha sido desarrollada en el seno del proyecto de investigación de excelencia titulado: «El patrimonio 
histórico de Agustín de Betancourt: estudio integral de las aportaciones a la ingeniería civil desde la ingeniería gráfica para su puesta en valor y difusión» (HAR2015-63503-P), financiado por el Ministerio de Economía y Competitividad dentro del Programa Estatal de Fomento de la Investigación Científica y Técnica de Excelencia, Subprograma Estatal de Generación del Conocimiento, en el marco del Plan Es- tatal de Investigación Científica y Técnica y de Innovación 2013-2016, y por el Fondo Europeo de Desarrollo Regional (FEDER). Asimismo, también agradecer muy sinceramente a la Fundación Canaria Orotava de Historia de la Ciencia por su amabilidad y disposición para la utilización del material de su website, así como a la Fundación Juanelo Turriano por la concesión de una de sus prestigiosas becas de doctorado.

\section{REFERENCIAS}

(1) Muñoz Bravo, J. (2008). Biografía cronológica de Don Agustín de Betancourt y Molina en el 250 aniversario de su nacimiento. Murcia: Acciona Infraestructuras.

(2) Bogoliúbov, A.N. (1973). Agustín de Betancourt: un héroe español del progreso. Madrid: Seminarios y Ediciones.

(3) Martín Medina, A. (2006). Agustín de Betancourt y Molina. Madrid: Dykinson.

(4) Padrón Acosta, S. (1958). El ingeniero Agustín de Béthencourt y Molina. La Laguna de Tenerife: Instituto de Estudios Canarios.

(5) Rumeu de Armas, A. (1968). Agustín de Betancourt, fundador de la Escuela de Caminos y Canales: nuevos datos biográficos. Madrid: Colegio Oficial de Ingenieros de Caminos, Canales y Puertos.

(6) Sáenz Ridruejo, F. (2016). Agustín de Betancourt, en España, Francia, Inglaterra y Rusia. En Cámara Muñoz, A. y Revuelta Pol, B. (Coord.), Libros, caminos y días: El viaje del ingeniero (pp. 95-123). Madrid: Fundación Juanelo Turriano.

(7) Cioranescu, A. (1965). Agustín de Betancourt: su obra técnica y científica. La Laguna de Tenerife: Instituto de Estudios Canarios.

(8) Rumeu de Armas, A. (1990). El Real Gabinete de Máquinas del Buen Retiro: origen, fundación y vicisitudes: una empresa técnica de Agustín de Betancourt. Madrid: Castalia-Fundación Juanelo Turriano.

(9) García Diego, J.A. (1986, 22 de septiembre). Agustín de Betancourt como espía industrial: estudios sobre la historia de la ciencia y de la técnica. En IV Congreso de la Sociedad Española de Historia de las Ciencias y de las Técnicas (pp. 105125). Valladolid: Consejería de Cultura y Bienestar Social de la Junta de Castilla y León.

(10) Proyecto Digital Betancourt. Disponible online: http://fundacionorotava.es/betancourt (último acceso 20/02/2017).

(11) Rojas-Sola, J.I., De la Morena-de la Fuente, E. (2016). Agustin de Betancourt's wind machine to drain marshy grounds: Approach to its geometric modeling with Autodesk Inventor Professional. Technologies, 5(1): 2, doi: http://dx.doi. org/10.3390/technologies5010002

(12) Villar-Ribera, R., Hernández-Abad, F., Rojas-Sola, J.I., Hernández-Díaz, D. (2011). Agustin de Betancourt’s telegraph: Study and virtual reconstruction. Mechanism and Machine Theory, 46(6): 820-830, doi: http://dx.doi.org/10.1016/j. mechmachtheory.2011.01.008

(13) Betancourt, A. (1789). Sur le nouveau moulin à vent. París: Institut National de France. Disponible online: http://fundacionorotava.es/pynakes/lise/betan_mouli_fr_01_18XX/2 (último acceso 20/02/2017).

(14) Shih, R.A. (2015). Parametric modeling with Autodesk Inventor 2016. Mission (Kansas, USA): SDC Publications.

(15) AENOR (1998). UNE-ENV 1991-2-4 Bases de proyecto y acciones en estructuras. Parte 2-4: Acciones en estructuras. Acciones del viento. Asociación Española de Normalización y Certificación (AENOR). 